\title{
STRUCTURAL THEOREMS FOR QUASIASYMPTOTICS OF DISTRIBUTIONS AT INFINITY
}

\author{
Jasson Vindas
}

\begin{abstract}
Complete structural theorems for quasiasymptotics of distributions are presented in this article. For this, asymptotically homogeneous functions and associate asymptotically homogeneous functions at infinity with respect to a slowly varying function are employed. The proposed analysis, based on the concept of asymptotically and associate asymptotically homogeneous functions, allows to obtain easier proofs of the structural theorems for quasiasymptotics at infinity in the so far only known case: when the degree of the quasiasymptotic is not a negative integer. Furthermore, new structural theorems for the case of negative integral degrees are obtained by this method.
\end{abstract}

\section{Introduction and Preliminaries}

The present article characterizes the quasiasymptotic behavior of Schwartz distributions at infinity. It continues my joint work with Pilipović $[\mathbf{1 5}]$ in which we have obtained complete structural theorems for the quasiasymptotic behavior of distributions at the origin.

We start this section by explaining the notation and concepts to be considered in this paper. The Schwartz spaces of test functions and distributions on the real line $\mathbb{R}$ are denoted by $\mathcal{D}$ and $\mathcal{D}^{\prime}$, respectively; the spaces of rapidly decreasing functions and its dual, the space of tempered distributions, are denoted by $\mathcal{S}$ and $\mathcal{S}^{\prime}$. We refer the reader to $[\mathbf{1 0}, \mathbf{1 7}, \mathbf{5}]$ for the properties of these spaces. Let us recall that a real-valued measurable function defined in some interval of the form $[A, \infty), A>0$, is called slowly varying function at infinity if $L$ is positive for large arguments and

$$
\lim _{x \rightarrow \infty} \frac{L(a x)}{L(x)}=1
$$

2000 Mathematics Subject Classification: Primary 41A60, 46F10; Secondary 42A24, 46F05, $46 \mathrm{~F} 99$.

Key words and phrases: Slowly varying functions, quasiasymptotics of distributions, almost homogeneous functions.

The author gratefully acknowledges support by the Louisiana State Board of Regents grant LEQSF(2005-2007)-ENH-TR-21. 
for any $a>0$. The standard reference for slowly varying functions is [11].

Let $L$ be slowly varying. We say that $f \in \mathcal{D}^{\prime}$ has quasiasymptotic behavior at infinity (has quasiasymptotic at infinity) in $\mathcal{D}^{\prime}$ with respect to $\lambda^{\alpha} L(\lambda), \alpha \in \mathbb{R}$, if for some $g \in \mathcal{D}^{\prime}$ and every $\phi \in \mathcal{D}$,

$$
\lim _{\lambda \rightarrow \infty}\left\langle\frac{f(\lambda x)}{\lambda^{\alpha} L(\lambda)}, \phi(x)\right\rangle=\langle g(x), \phi(x)\rangle .
$$

If (1.1) holds, we also say that $f$ has quasiasymptotic of degree $\alpha$ at infinity with respect to the slowly varying function $L$. Because of [11, Lemma 1.2], the function $\mathrm{L}$ can be assumed to be positive and defined on $[0, \infty)$. The quasiasymptotic of distributions at the origin is defined in a similar way. The last definition was introduced by Pilipović in $[\mathbf{8}]$ and $[\mathbf{9}]$, where it is assumed $g \neq 0$, but we extend the definition by allowing $g$ to be 0 . The quasiasymptotic behavior was originally defined by Zavialov in [18] for distributions with support bounded at the left, so due to historical reasons, it is also usual in the literature to define the quasiasymptotic at infinity only for tempered distributions with support bounded on one side $[\mathbf{1 8}, \mathbf{1 7}]$; the second reason for this is, probably, that in the case when the degree of the quasiasymptotic is not an negative integer, one has a decomposition theorem $[\mathbf{1 7}$, p.134] that allows to reduce the two sided case to the one sided case. However, the most interesting results of this article are concerned with the negative integral degree case.

We also express (1.1), a more convenient notation for our purposes, by saying

$$
f(\lambda x)=\lambda^{\alpha} L(\lambda) g(x)+o\left(\lambda^{\alpha} L(\lambda)\right) \quad \text { as } \lambda \rightarrow \infty \text { in } \mathcal{D}^{\prime},
$$

which should always be interpreted in the weak topology of $\mathcal{D}^{\prime}$, i.e., in the sense of (1.1).

It is easy to prove (see [5, p. 161], [9] and [17]) that (1.2) forces $g$ to be homogeneous with the degree of homogeneity $\alpha$. Since we know explicitly all homogeneous distributions on the real line [5, p.72], then either $g$ has the form

$$
g(x)=C_{-} x_{-}^{\alpha}+C_{+} x_{+}^{\alpha}, \quad \text { if } \alpha \notin\{-1,-2,-3, \ldots\},
$$

for some constants $C_{-}$and $C_{+}$, or

$$
g(x)=\gamma \delta^{(k-1)}(x)+\beta x^{-k}, \quad \text { if } \alpha=-k \in\{-1,-2,-3, \ldots\},
$$

for some constants $\gamma$ and $\beta$, where here we are following the notation from $[\mathbf{5}$, Chapter 2]. Other particular distributions used in this article are the Heaviside function $H(x)$ and the distributions $\operatorname{Pf}\left(H(x) / x^{k}\right)$, for $k \in \mathbb{N}$, they are also defined in [5, Chapter 2].

The quasiasymptotic of distributions (1.2) has been characterized when $\alpha \notin$ $\{-1,-2, \ldots\}$. Indeed, one has the following structural theorem [9, Theorem 4] (see also $\left[\mathbf{1 7}\right.$, p. 134]). Let $f \in \mathcal{D}^{\prime}$ have the quasiasymptotic behavior at infinity in $\mathcal{D}^{\prime}$,

$$
f(\lambda x)=C_{-} L(\lambda) \frac{(\lambda x)_{-}^{\alpha}}{\Gamma(\alpha+1)}+C_{+} L(\lambda) \frac{(\lambda x)_{+}^{\alpha}}{\Gamma(\alpha+1)}+o\left(\lambda^{\alpha} L(\lambda)\right), \quad \lambda \rightarrow \infty,
$$


$\alpha \notin\{-1,-2, \ldots\}$, then there exist a non-negative integer $m$ and an $m$-primitive $F$ of $f$, that is $F^{(m)}=f$, such that $F$ is continuous and

$$
\lim _{x \rightarrow \pm \infty} \frac{\Gamma(\alpha+m+1) F(x)}{x^{m}|x|^{\alpha} L(|x|)}=C_{ \pm} .
$$

As a consequence of our analysis in terms of asymptotically homogeneous functions, we will give a new proof of this theorem in Section 2. Furthermore, in Section 3 , we will complete the characterization of quasiasymptotics of Schwartz distributions by obtaining the structural theorem for negative integral degrees, it will be the main result of this paper.

Suppose $f \in \mathcal{D}^{\prime}$ has quasiasymptotic in $\mathcal{D}^{\prime}$, then this condition implies that $f$ is a tempered distribution $[\mathbf{8 , 9}, \mathbf{5}]$. If we replace the space $\mathcal{D}$ by $\mathcal{S}$ in $(1.1)$, then we say that $f$ has quasiasymptotic at infinity with respect to $\lambda^{\alpha} L(\lambda)$ in $\mathcal{S}^{\prime}$. When the degree of the quasiasymptotic is not a negative integer, Pilipović has shown in $[8$, Theorem 4] that the existence of the quasiasymptotic in $\mathcal{D}^{\prime}$ implies the existence of the quasiasymptotic in $\mathcal{S}^{\prime}$. In Section 3 , this result is extended to negative integral degrees as a direct consequence of the structural theorem, Theorem 3.3. This fact is stated in Remark 3.1. Further related questions are discussed in Section 4.

\section{Asymptotically Homogeneous Functions}

In this section two classes of functions of regular asymptotic behavior at infinity are discussed, the class of asymptotically homogeneous functions at infinity and the class of associate asymptotically homogeneous functions of degree 0 at infinity. These functions are the analog to asymptotically homogeneous functions and associate asymptotically homogeneous functions at the origin with respect to a slowly varying function [15]. We will later derive the announced structural theorems for quasiasymptotics at infinity from the fundamental properties of these classes of functions. The technique to be used here is based in the analysis of the parametric coefficients resulting after performing several integrations of the quasiasymptotic. This has been previously applied in $[4, \mathbf{1 2}, \mathbf{1 3}, \mathbf{1 4}]$ to the study of pointwise Fourier inversion formulas for distributional point values and jump behavior of distributions; furthermore, in [15], asymptotically homogeneous functions have been effectively used to obtain a complete characterization for quasiasymptotics of Schwartz distributions at the origin. Let us proceed to define asymptotically homogeneous functions at infinity.

DeFinition 2.1. A function $b$ is said to be asymptotically homogeneous of degree $\alpha$ at infinity with respect to the slowly varying function $L$, if it is measurable and defined in some interval $[A, \infty), A>0$, and for each $a>0$,

$$
b(a x)=a^{\alpha} b(x)+o(L(x)), \quad x \rightarrow \infty .
$$

In the following, some of the fundamental properties of asymptotically homogeneous functions at infinity are stated and discussed. As previously mentioned, the author and Pilipović has introduced and studied the class of asymptotically homogeneous functions at the origin in the cited article. In fact, one has that asymptotically homogeneous functions at infinity are of the form $b(x)=c(1 / x)$ where $c$ is 
asymptotically homogeneous at the origin, and hence most of the properties of this class of functions can be obtained by reducing to those of asymptotically homogeneous functions at the origin by the change of variables $x \leftrightarrow x^{-1}$ and, obviously, the same holds in the opposite direction.

Concerning to the next following three results, Theorem 2.1, Lemma 2.1 and Theorem 2.2, the proofs have been obtained for asymptotically homogeneous functions at the origin in [15, Section 3], and will be therefore omitted here. Before going to the statements, the author would like to make some comments. I learned recently from [11, Section 2.4] that the mentioned results could be also obtained from properties of a class of functions studied by Bojanić and Karamata in [2], but, at the time we wrote [15], we were not aware of the existence of such results. This class of functions has been studied in $[\mathbf{1}, \mathbf{1 1}]$ as well. The functions introduced by Bojanić and Karamata are measurable functions defined in some interval of the form $[A, \infty), A>0$, satisfying

$$
c(a x)=c(x)+\tau(a) x^{\alpha} L(x)+o\left(x^{\alpha} L(x)\right), \quad x \rightarrow \infty ;
$$

so if $b$ is asymptotically homogeneous at infinity of degree $\alpha$ with respect to $L$, then $c(x)=b(x) / x^{\alpha}$ satisfies $(2.1)$ with $\tau(a)=0$ and $\alpha$ replaced by $-\alpha$. So, Theorem 2.1, Lemma 2.1 and Theorem 2.2 are consequences of the results from [11, Section 2.4] as well.

The properties of asymptotically homogeneous functions at infinity are summarized in the following results. The first theorem estimates the behavior of such functions at infinity when the degree is negative.

THEOREM 2.1. Let $b$ be asymptotically homogeneous of degree $\alpha<0$ at infinity with respect to the slowly varying function $L$. Then $b(x)=o(L(x)), x \rightarrow \infty$.

Note that in Definition 2.1 no uniformity with respect to $a$ is assumed; however, we have the following lemma.

LEMMA 2.1. Let $b$ be an asymptotically homogeneous function of degree $\alpha$ at infinity with respect to $L$. Then, the relation $b(a x)=a^{\alpha} b(x)+o(L(x)), x \rightarrow \infty$, holds uniformly for a in compact subsets of $(0, \infty)$.

The next theorem explores the asymptotic behavior of asymptotically homogeneous functions of positive degree.

THEOREM 2.2. Suppose that $b$ is asymptotically homogeneous of degree $\alpha>0$ at infinity with respect to the slowly varying function $L$. Then, there exists a number $\gamma$ such that,

$$
b(x)=\gamma x^{\alpha}+o(L(x)), \quad x \rightarrow \infty .
$$

In particular, for each $\sigma>0$ we have $b(x)=\gamma x^{\alpha}+o\left(x^{\sigma}\right), x \rightarrow \infty$.

Notice that (2.2) trivially implies that $b$ is asymptotically homogeneous of degree $\alpha$ with respect to $L$.

We now make the link between asymptotically homogeneous functions and quasiasymptotic behavior of distributions. The proof of the next proposition is analogous to that of [15, Proposition 3.1]. 
Proposition 2.1. Let $f \in \mathcal{D}^{\prime}$ have quasiasymptotic behavior at infinity

$$
f(\lambda x)=L(\lambda) g(\lambda x)+o\left(\lambda^{\alpha} L(\lambda)\right) \quad \text { as } \lambda \rightarrow \infty \text { in } \mathcal{D}^{\prime},
$$

where $L$ is a slowly varying function and $g$ is a homogeneous distribution of degree $\alpha \in \mathbb{R}$. Let $n \in \mathbb{N}$. Suppose that $g$ admits a primitive of order $n$, that is $G_{n} \in \mathcal{D}^{\prime}$ and $G_{n}^{(n)}=g$, which is homogeneous of degree $n+\alpha$. Then, for any given $F_{n}$, an n-primitive of $f$ in $\mathcal{D}^{\prime}$, there exist functions $b_{0}, \ldots, b_{n-1}$, continuous on $(0, \infty)$, such that

$$
F_{n}(\lambda x)=L(\lambda) G_{n}(\lambda x)+\sum_{j=0}^{n-1} \lambda^{\alpha+n} b_{j}(\lambda) \frac{x^{n-1-j}}{(n-1-j) !}+o\left(\lambda^{\alpha+n} L(\lambda)\right)
$$

as $\lambda \rightarrow \infty$ in $\mathcal{D}^{\prime}$, where each $b_{j}$ is asymptotically homogeneous of degree $-\alpha-j-1$.

Proof. Relation (2.4) follows from (2.3) and the definitions of convergence in $\mathcal{D}^{\prime}$ and primitives in $\mathcal{D}^{\prime}$ (see [15, Proposition 3.1] for a complete argument).

Thus we shall concentrate in showing the property of the $b_{j}$ 's. We set $F_{m}=$ $F_{n}^{(n-m)}$ and $G_{m}=G_{n}^{(n-m)}, m \in\{1, \ldots, n\}$. By differentiating relation $(2.4)(n-$ $m$ )-times, it follows that

$$
F_{m}(\lambda x)=L(\lambda) G_{m}(\lambda x)+\sum_{j=0}^{m-1} \lambda^{\alpha+m} b_{j}(\lambda) \frac{x^{m-1-j}}{(m-1-j) !}+o\left(\lambda^{\alpha+m} L(\lambda)\right)
$$

as $\lambda \rightarrow \infty$ in $\mathcal{D}^{\prime}$. Choose $\phi \in \mathcal{D}$ such that $\int_{-\infty}^{\infty} \phi(x) x^{j} d x=0$ for $j=1, \ldots, m-1$, and $\int_{-\infty}^{\infty} \phi(x) d x=1$. Then evaluating (2.5) at $\phi$, we have that as $\lambda \rightarrow \infty$

$$
\begin{aligned}
& (a \lambda)^{\alpha+m} b_{m-1}(a \lambda)+L(a \lambda)\left\langle G_{m}(a \lambda x), \phi(x)\right\rangle+o\left(\lambda^{\alpha+m} L(\lambda)\right) \\
& =\left\langle F_{m}(a \lambda x), \phi(x)\right\rangle=\frac{1}{a}\left\langle F_{m}(\lambda x), \phi\left(\frac{x}{a}\right)\right\rangle \\
& =\lambda^{\alpha+m} b_{m-1}(\lambda)+L(\lambda)\left\langle G_{m}(a \lambda x), \phi(x)\right\rangle+o\left(\lambda^{\alpha+m} L(\lambda)\right),
\end{aligned}
$$

and so, with $j=m-1 \in\{0, \ldots, n-1\}$, for each $a>0$,

$$
b_{j}(a \lambda)=a^{-\alpha-j-1} b_{j}(\lambda)+o(L(\lambda)), \lambda \rightarrow \infty .
$$

Suppose now that $f \in \mathcal{D}^{\prime}$ is so that

$$
f(\lambda x)=C_{-} L(\lambda) \frac{(\lambda x)_{-}^{\alpha}}{\Gamma(\alpha+1)}+C_{+} L(\lambda) \frac{(\lambda x)_{+}^{\alpha}}{\Gamma(\alpha+1)}+o\left(\lambda^{\alpha} L(\lambda)\right) \quad \text { as } \lambda \rightarrow \infty \text { in } \mathcal{D}^{\prime},
$$

where $\alpha \notin\{-1,-2, \ldots\}$. Then, on combining Proposition 2.1, Theorem 2.1, and Theorem 2.2, one obtains that for each $n \in \mathbb{N}$ and $F_{n}$, an $n$-primitive of $f$, there exist constants $\gamma_{0}, \ldots, \gamma_{n-1}$ such that in the sense of convergence in $\mathcal{D}^{\prime}$,

$F_{n}(\lambda x)=\sum_{j=0}^{n-1} \gamma_{j} \frac{(\lambda x)^{j}}{j !}+C_{-} \frac{(-1)^{n} L(\lambda)(\lambda x)_{-}^{\alpha+n}}{\Gamma(\alpha+n+1)}+C_{+} \frac{L(\lambda)(\lambda x)_{+}^{\alpha+n}}{\Gamma(\alpha+n+1)}+o\left(\lambda^{\alpha+n} L(\lambda)\right)$

as $\lambda \rightarrow \infty$. This produces a new proof of the structural theorem from $[\mathbf{9}]$ mentioned at the introduction. 
THEOREM 2.3. Let $f \in \mathcal{D}^{\prime}$ have quasiasymptotic behavior at infinity in $\mathcal{D}^{\prime}$,

$$
f(\lambda x)=C_{-} L(\lambda) \frac{(\lambda x)_{-}^{\alpha}}{\Gamma(\alpha+1)}+C_{+} L(\lambda) \frac{(\lambda x)_{+}^{\alpha}}{\Gamma(\alpha+1)}+o\left(\lambda^{\alpha} L(\lambda)\right), \quad \lambda \rightarrow \infty .
$$

If $\alpha \notin\{-1,-2, \ldots\}$, then there exist a non-negative integer $m>-\alpha-1$ and an $m$-primitive $F$ of $f$ such that $F$ is continuous and

$$
\lim _{x \rightarrow \pm \infty} \frac{\Gamma(\alpha+m+1) F(x)}{x^{m}|x|^{\alpha} L(|x|)}=C_{ \pm} .
$$

Conversely, if these conditions hold, then (by differentiation) (2.7) follows.

Proof. It follows from the definition of convergence in $\mathcal{D}^{\prime}$ that there is $m \in \mathbb{N}$ such that any $m$-primitive of $f$ is continuous and (2.6) holds (with $n=m$ ) uniformly for $x \in[-1,1]$. Pick a specific $m$-primitive of $f$, say $F_{m}$, then from $(2.6)$ there is a polynomial $p$ of degree at most $m-1$ such that

$F_{m}(\lambda x)=p(\lambda x)+C_{-} L(\lambda) \frac{(-1)^{m}(\lambda x)_{-}^{\alpha+m}}{\Gamma(\alpha+m+1)}+C_{+} L(\lambda) \frac{(\lambda x)_{+}^{\alpha+m}}{\Gamma(\alpha+m+1)}+o\left(\lambda^{\alpha+m} L(\lambda)\right)$ as $\lambda \rightarrow \infty$, uniformly for $x \in[-1,1]$. Then setting $F=F_{m}-p, x=1,-1$ and replacing $\lambda$ by $x$, relation (2.8) follows at once.

REMARK 2.1. Since $p_{m-1}(x)=o\left(x^{m+\alpha} L(x)\right) \rightarrow 0, x \rightarrow \infty$, whenever $\alpha>-1$, we have that in such a case the polynomial is irrelevant in the proof of the last Theorem. Hence in fact (2.8) holds for every $m$-primitive of $f$, provided that $\alpha>-1$.

REMARK 2.2. We obtain at once the decomposition theorem from [17, p.134].

We now give a second application of asymptotically homogeneous functions, here we turn our attention to quasiasymptotics whose degrees are negative integers.

Proposition 2.2. Let $f \in \mathcal{D}^{\prime}$ have the quasiasymptotic behavior at infinity

$$
f(\lambda x)=L(\lambda) g(\lambda x)+o\left(\lambda^{-k} L(\lambda)\right) \quad \text { as } \lambda \rightarrow \infty \text { in } \mathcal{D}^{\prime},
$$

where $k \in\{2,3, \ldots\}$ and $g$ is a homogeneous distribution of degree $-k$. Let $G$ be a homogeneous distribution of degree -1 such that $G^{(k-1)}=g$. Then for some $(k-1)$-primitive of $f, F_{k-1}$, we have that

$$
F_{k-1}(\lambda x)=L(\lambda) G(\lambda x)+o\left(\lambda^{-1} L(\lambda)\right) \quad \text { as } \lambda \rightarrow \infty \text { in } \mathcal{D}^{\prime} .
$$

Conversely, relation (2.10) implies (2.9).

Proof. It follows directly from Proposition 2.1 and Theorem 2.2.

Proposition 2.2 reduces our study to the case of quasiasymptotics of degree -1 which we shall postpone for the next section. We now set the ground for the next section. What makes impossible the application of Proposition 2.1 to the -1 degree case is the fact that in general we cannot single out homogeneous distributions from the primitives of a homogeneous distribution of degree -1 . In Section 3, the technique of integrating the quasiasymptotic and studying the coefficients of integration is employed again; moreover, the main coefficient of this integration 
will fit into the context of associate asymptotically homogeneous functions, which we now define.

Definition 2.2. A function $b$ is said to be associate asymptotically homogeneous of degree 0 at infinity with respect to the slowly varying function $L$, if it is measurable and defined in some interval $[A, \infty), A>0$, and for each $a>0$,

$$
b(a x)=b(x)+\beta \log a L(x)+o(L(x)), \quad x \rightarrow \infty,
$$

for some constant $\beta$.

Note that the property (2.11) is exactly (2.1) with $\alpha=0$ and $\tau(a)=\beta \log a$; indeed, when $\alpha=0$, it can be shown [11, Theorem 2.9] that (2.1) forces $\tau$ to have this form. It can be also shown by using a classical argument of Korevaar, van Aardenne Ehrenfest and de Bruijn that (2.11) must hold uniformly for $a$ on compact sets (for the usage of this argument see $[\mathbf{6}],[\mathbf{1 1}$, Theorem 2.12] and $[\mathbf{1 5}$, Lemma 3.5]).

Using Theorem 2.1, we can roughly estimate the behavior of associate asymptotically homogeneous functions of degree 0 at infinity.

Proposition 2.3. Let $b$ be associate asymptotically homogeneous of degree 0 at infinity with respect to $L$, then for each $\sigma>0$,

$$
b(x)=o\left(x^{\sigma}\right), \quad x \rightarrow \infty .
$$

Proof. It is known that $L(x)=o\left(x^{\sigma}\right)$, for each $\sigma>0[\mathbf{1 1}]$. Hence $b(a x)=$ $b(x)+o\left(x^{\sigma}\right)$ and thus $x^{-\sigma} b(x)$ is asymptotically homogeneous of degree $-\sigma$ with respect to $L \equiv 1$, so (2.12) follows from Theorem 2.1.

The next theorem will be very important in the next section. Recall that $H$ denotes the Heaviside function, i.e., the characteristic function of $(0, \infty)$.

THEOREM 2.4. Let $b$ be a locally integrable associate asymptotically homogeneous function of degree zero at infinity with respect to the slowly varying function L. Suppose that $b$ is defined on $[A, \infty)$. Then

$$
b(\lambda x) H(\lambda x-A)=b(\lambda) H(x)+L(\lambda) \beta \log x H(x)+o(L(\lambda))
$$

as $\lambda \rightarrow \infty$ in $\mathcal{S}^{\prime}$, where $H$ is the Heaviside function.

Proof. Let $\lambda_{0}$ be any positive number. The function $b$ can be decomposed as $b=b_{1}+b_{2}$, where $b_{1} \in L^{1}(\mathbb{R})$ has compact support and $b_{2}(x)=b(x) H\left(x-\lambda_{0}\right)$ is associate asymptotically homogeneous function of degree zero at infinity. Since $b_{1}$ satisfies the moment asymptotic expansion [5], it follows that $b_{1}(\lambda x)=O\left(\lambda^{-1}\right)=$ $o(L(\lambda))$ as $\lambda \rightarrow \infty$ in $\mathcal{S}^{\prime}$. Therefore, we can always assume that $A=\lambda_{0}$, where $\lambda_{0}$ is selected at our convenience.

Our aim is to show that there is some $\lambda_{0}>1$ such that

$$
J(x, \lambda):=\phi(x) \frac{b(\lambda x)-b(\lambda)-\beta L(\lambda) \log x}{L(\lambda)} H\left(\lambda x-\lambda_{0}\right)
$$


is dominated by an integrable function, whenever $\phi \in \mathcal{S}$, for the use of the Lebesgue dominated convergence theorem. For this goal, we can always assume that $L$ is positive everywhere and satisfies the following estimate (see for example the arguments given in [15, Section 2]),

$$
\frac{L(\lambda x)}{L(\lambda)} \leqslant M \max \left\{x^{-1 / 4}, x^{1 / 4}\right\}, \quad x, \lambda \in(0, \infty),
$$

for some positive constant $M$. Because of the uniformity of (2.11) on compact sets, there exists a $\lambda_{0}>1$ such that $|b(\lambda x)-b(\lambda)-\beta L(\lambda) \log x|<L(\lambda), x \in[1,2]$, $\lambda_{0}<\lambda$. Let $n$ be a positive integer. We keep $\lambda_{0}<\lambda$ and $x \in\left[2^{n}, 2^{n+1}\right]$. Then

$$
\begin{aligned}
|b(\lambda x)-b(\lambda)-\beta L(\lambda) \log x| \leqslant|b(\lambda x)-b(\lambda)|+|\beta| L(\lambda) \log x \\
\leqslant|\beta| L(\lambda) \log x+|b(2(\lambda x / 2))-b(\lambda x / 2)-\beta L(\lambda x / 2) \log 2| \\
\quad+|\beta| L(\lambda x / 2) \log 2+|b(\lambda x / 2)-b(\lambda)| \\
\quad \leqslant|\beta| L(\lambda) \log x+(1+|\beta| \log 2) L(\lambda x / 2)+|b(\lambda x / 2)-b(\lambda)| \\
\leqslant(1+|\beta| \log 2) \sum_{j=1}^{n} L\left(2^{-j} \lambda x\right)+|\beta| L(\lambda) \log 2 x+L(\lambda) \\
\leqslant\left(M x^{1 / 4}(1+|\beta| \log 2) \sum_{j=1}^{n}(1 / 2)^{j / 4}+|\beta| \log 2 x+1\right) L(\lambda)
\end{aligned}
$$

where the last inequality follows from (2.14). So if $\lambda_{0}<\lambda$ and $1<x$, then

$$
\left|\frac{b(\lambda x)-b(\lambda)-\beta L(\lambda) \log x}{L(\lambda)}\right| \leqslant M_{1} x^{1 / 4},
$$

for some $M_{1}>0$. Now if $\lambda_{0} / \lambda<x<1$, we have that

$$
\begin{aligned}
& \left|\frac{b(\lambda x)-b(\lambda)-\beta L(\lambda) \log x}{L(\lambda)}\right| \\
& \quad \leqslant\left(1+\frac{L(\lambda x)}{L(\lambda)}\right)|\beta \log x|+\left|\frac{b(\lambda)-b(\lambda x)-\beta L(\lambda x) \log x^{-1}}{L(\lambda)}\right| \\
& \quad \leqslant\left(1+M x^{-1 / 4}\right)|\beta \log x|+\frac{L(\lambda x)}{L(\lambda)}\left|\frac{b\left(\lambda x\left(x^{-1}\right)\right)-b(\lambda x)-\beta L(\lambda x) \log x^{-1}}{L(\lambda x)}\right| \\
& \quad \leqslant\left(1+M x^{-1 / 4}\right)|\beta \log x|+M M_{1} x^{-1 / 2} .
\end{aligned}
$$

Therefore $J(x, \lambda)$ is dominated by an integrable function for $\lambda>\lambda_{0}$, so we apply Lebesgue dominated convergence theorem to deduce that $\lim _{\lambda \rightarrow \infty} \int_{0}^{\infty} J(x, \lambda) d x=0$. Finally,

$$
\begin{aligned}
\langle b(\lambda x) H(\lambda x & \left.\left.-\lambda_{0}\right), \phi(x)\right\rangle-b(\lambda) \int_{0}^{\infty} \phi(x) d x-\beta L(\lambda) \int_{0}^{\infty} \log x \phi(x) d x \\
& =\int_{\lambda_{0} / \lambda}^{\infty} b(\lambda x) \phi(x) d x-b(\lambda) \int_{0}^{\infty} \phi(x) d x-\beta L(\lambda) \int_{0}^{\infty} \log x \phi(x) d x \\
& =L(\lambda) \int_{0}^{\infty} J(x, \lambda) d x+L(\lambda) O\left(\frac{\log \lambda}{\lambda}\right)+O\left(\frac{b(\lambda)}{\lambda}\right)
\end{aligned}
$$




$$
=o(L(\lambda))+L(\lambda) O\left(\frac{b(\lambda)}{\lambda L(\lambda)}\right)=o(L(\lambda)), \quad \lambda \rightarrow \infty
$$

where in the last equality we have used Proposition 2.3 and the fact that slowly varying functions are $o\left(\lambda^{\sigma}\right)$ for any $\sigma>0[\mathbf{1 1}]$. This completes the proof of (2.13).

COROLlARY 2.1. Let b be an associate asymptotically homogeneous function of degree 0 at infinity with respect to $L$. Then, there exists an associate asymptotically homogeneous function $c \in C^{\infty}[0, \infty)$ such that $b(x)=c(x)+o(L(x))$ as $x \rightarrow \infty$.

Proof. We may assume that $L \in C^{\infty}[0, \infty)[\mathbf{1 1}$, Section 1.4]. Find $B$ such that $b$ is locally bounded in $[B, \infty)$, this can be done because of Proposition 2.3. Take $\phi \in \mathcal{D}$ such that $\int_{0}^{\infty} \phi(t) d t=1$ and set

$$
c(x)=\int_{B / x}^{\infty} b(x t) \phi(t) d t-\beta L(x) \int_{0}^{\infty} \phi(t) \log t d t,
$$

the corollary now follows from Theorem 2.4.

We may also use Corollary 2.1 to obtain a representation formula for associate asymptotically homogeneous functions, this is the analog to [11, Theorem 1.2] for slowly varying functions.

THEOREM 2.5. The function $b$ is associate asymptotically homogeneous of degree 0 at $\infty$ satisfying (2.11) if and only if there is a positive number $A$ such that

$$
b(x)=\eta(x)+\int_{A}^{x} \tau(t) \frac{L(t)}{t} d t, \quad x \geqslant A,
$$

where $\eta$ is a locally bounded measurable function on $[A, \infty)$ such that $\eta(x)=M+$ $o(L(x))$ as $x \rightarrow \infty$, for some number $M$, and $\tau$ is a $C^{\infty}$-function such that $\tau(x)=$ $\beta+o(1)$ as $x \rightarrow \infty$.

Proof. Assume first that $b_{1}$ is $C^{\infty}$, defined on $[0, \infty)$ and satisfies that hypothesis of the theorem. We can find $L_{1} \sim L$ which is $C^{\infty}$ and satisfies $x L_{1}^{\prime}(x)=o(L(x))$ as $x \rightarrow \infty[\mathbf{1 1}, \mathrm{p} .7]$. Let $\phi$ and $c$ as in the proof of Corollary 2.1 corresponding to $b_{1}$ and $L_{1}$, additionally assume that $\operatorname{supp} \phi \subseteq(0, \infty)$. From Theorem 2.4 , we have that

$$
b_{1}^{\prime}(\lambda x)=\frac{b_{1}(\lambda)}{\lambda} \delta(x)+\beta \frac{L(\lambda)}{\lambda} \operatorname{Pf}\left(\frac{H(x)}{x}\right)+o\left(\frac{L(\lambda)}{\lambda}\right) \quad \text { as } \lambda \rightarrow \infty
$$

in $\mathcal{S}^{\prime}$, since distributional asymptotics can be differentiated. Then, for $x$ positive

$$
\begin{aligned}
x c^{\prime}(x) & =x \int_{0}^{\infty} b_{1}^{\prime}(x t) t \phi(t) d t-\beta x L_{1}^{\prime}(x) \int_{0}^{\infty} \phi(t) \log t d t \\
& =x \int_{0}^{\infty} b_{1}^{\prime}(x t) t \phi(t) d t+o(L(x)) \\
& =b_{1}(x) \cdot 0+\beta L(x) \int_{0}^{\infty} \phi(t) d t+o(L(x)) \\
& =\beta L(x)+o(L(x)) \text { as } x \rightarrow \infty .
\end{aligned}
$$


Set $\tau(x)=x c^{\prime}(x) / L(x)$. Find $A>0$ such that $L$ is locally integrable on $[A, \infty)$, one has that $b_{1}(x)=c(A)+\int_{A}^{x} \tau(t)(L(t) / t) d t+o(L(x))$.

In the general case, let $A$ be a number such that $b$ and $L$ are locally bounded on $[A, \infty)$ and let $b_{1}$ the function from Corollary 2.1 such that $b(x)=b_{1}(x)+o(L(x))$, then we can apply the previous argument to $b_{1}$ to find $\tau$ as before, so we obtain $(2.15)$ with $\eta(x)=b(x)-\int_{A}^{x} \tau(t)(L(t) / t) \mathrm{d} t=c(A)+o(L(x))$.

REMARK 2.3. A slightly different representation formula is given in $[\mathbf{1 1}$, Theorem 2.13], but, except for the smoothness of $\tau$, both are equivalent.

\section{Quasiasymptotic of Negative Integral Degree}

This section will be dedicated to the quasiasymptotic with negative integral degree. Because of Proposition 2.2, we start with case of degree -1 .

We should introduce some notation that will be needed. In the following for all $n \in \mathbb{N}$ we denote by $l_{n}$ the primitive of $\log |x|$ with the property that $l_{n}(0)=0$ and $l_{n}^{\prime}=l_{n-1}$. We have an explicit formula for them:

$$
l_{n}(x)=\frac{x^{n}}{n !} \log |x|-\frac{x^{n}}{n !} \sum_{j=1}^{n} \frac{1}{j}, \quad x \in \mathbb{R},
$$

which can be easily verified by direct differentiation. They satisfy

$$
l_{n}(a x)=a^{n} l_{n}(x)+\frac{(a x)^{n}}{n !} \log a, \quad a>0 .
$$

THEOREM 3.1. Let $f \in \mathcal{D}^{\prime}$ have quasiasymptotic at infinity of the form

$$
f(\lambda x)=\gamma \lambda^{-1} L(\lambda) \delta(x)+\beta \lambda^{-1} L(\lambda) x^{-1}+o\left(\lambda^{-1} L(\lambda)\right) \quad \text { as } \lambda \rightarrow \infty \text { in } \mathcal{D}^{\prime} .
$$

For each $n \in \mathbb{N}$, choose an n-primitive $F_{n}$ of $f$ such that $F_{n}^{\prime}=F_{n-1}$. Then, there exists an associate asymptotically homogeneous function b satisfying

$$
b(a x)=b(x)+\beta \log a L(x)+o(L(x), \quad x \rightarrow \infty,
$$

such that for any $n$ the distribution $F_{n+1}$ satisfies

$$
F_{n+1}(\lambda x)=b(\lambda) \frac{(\lambda x)^{n}}{n !}+\gamma L(\lambda) \frac{(\lambda x)^{n}}{2 n !} \operatorname{sgn} x+\beta L(\lambda) \lambda^{n} l_{n}(x)+o\left(\lambda^{n} L(\lambda)\right)
$$

as $\lambda \rightarrow \infty$, in the sense of convergence in $\mathcal{D}^{\prime}$. Moreover, there exists $n_{0} \in \mathbb{N}$ such that for all $n \geqslant n_{0}$ the distribution $F_{n+1}$ is a continuous function and (3.4) holds uniformly for $x \in[-1,1]$. In particular, for $n \geqslant n_{0}$ one has that

$$
F_{n+1}(x)=b(|x|) \frac{x^{n}}{n !}+\gamma \frac{x^{n}}{2 n !} L(|x|) \operatorname{sgn} x-\beta L(|x|) \frac{x^{n}}{n !} \sum_{j=1}^{n} \frac{1}{j}+o\left(|x|^{n} L(|x|)\right)
$$

as $x \rightarrow \pm \infty$, in the ordinary sense. Conversely, it follows from Theorem 2.4 that relation (3.5) implies (3.4), and (by differentiation) (3.2) follows. 
Proof. We shall study, as we have been doing, the coefficients of the integration of (3.2). We now proceed to integrate (3.2) once, so we obtain

$$
F_{1}(\lambda x)=b(\lambda)+\frac{\gamma}{2} L(\lambda) \operatorname{sgn} x+\beta L(\lambda) \log |x|+o(L(\lambda)) \quad \text { as } \lambda \rightarrow \infty
$$

in $\mathcal{D}^{\prime}$. Now, using the standard trick of evaluating at $\phi \in \mathcal{D}$ with the property $\int_{-\infty}^{\infty} \phi(x) d x=1$, one obtains that

$$
\begin{aligned}
& b(\lambda a)+\frac{\gamma}{2} L(\lambda a) \int_{-\infty}^{\infty} \operatorname{sgn} x \phi(x) d x+\beta L(\lambda a) \int_{-\infty}^{\infty} \log |x| \phi(x) d x+o(L(\lambda)) \\
& =\left\langle F_{1}(\lambda a x), \phi(x)\right\rangle=\frac{1}{a}\left\langle F_{1}(\lambda x), \phi\left(\frac{x}{a}\right)\right\rangle \\
& =b(\lambda)+\frac{\gamma}{2} L(\lambda) \int_{-\infty}^{\infty} \operatorname{sgn} x \phi(x) d x+\beta L(\lambda) \int_{-\infty}^{\infty} \log |a x| \phi(x) d x+o(L(\lambda)),
\end{aligned}
$$

$\lambda \rightarrow \infty$, for each $a>0$. So, we see that $b$ satisfies (3.3) for each $a>0$. Further integration of (3.6) gives,

$$
\begin{aligned}
F_{n+1}(\lambda x)=b(\lambda) \frac{(\lambda x)^{n}}{n !} & +\sum_{j=1}^{n} \lambda^{n} b_{j}(\lambda) \frac{x^{n-j}}{(n-j) !}+\gamma L(\lambda) \operatorname{sgn} x \frac{(\lambda x)^{n}}{2 n !} \\
& +\beta L(\lambda) \lambda^{n} l_{n}(x)+o\left(\lambda^{n} L(\lambda)\right) \text { as } \lambda \rightarrow \infty \text { in } \mathcal{D}^{\prime}
\end{aligned}
$$

As in the proof of Proposition 2.1, one shows that the $b_{j}$ 's are asymptotically homogeneous functions of degree $-j$ with respect to $L$, hence (3.4) follows from Theorem 2.1 applied to the $b_{j}$ 's. The next assertion follows from the definition of convergence in $\mathcal{D}^{\prime}$. Relation (3.5) is shown by making $x= \pm 1$ in (3.4) and then changing $\lambda \leftrightarrow x$. Finally, since only the behavior of $b$ at infinity plays a roll in (3.5), we may assume that $b$ is locally integrable, so the converse is obtained after application of Theorem 2.4 and [8, Theorem 3].

Theorem 3.1 is a structural theorem, but we shall give a version free of $b$.

THEOREM 3.2. Let $f \in \mathcal{D}^{\prime}$. Then $f$ has quasiasymptotic at infinity of the form (3.2) if and only if there exists an $(m+1)$-primitive $F$ of $f$, continuous, such that for each $a>0$,

$$
\lim _{x \rightarrow \infty} \frac{m !\left(a^{-m} F(a x)-(-1)^{m} F(-x)\right)}{x^{m} L(x)}=\gamma+\beta \log a .
$$

Proof. The limit (3.7) follows from (3.5), (3.3) and (3.1) by direct computation. For the converse, rewrite $(3.7)$ as

$$
a^{-m} F(a x)-(-1)^{m} F(-x)=(\gamma+\beta \log a) \frac{x^{m}}{m !} L(x)+o\left(x^{m} L(x)\right), \quad x \rightarrow \infty,
$$

for each $a>0$. Set

$$
b(x)=m ! x^{-m} F(x)-\left(\frac{\gamma}{2}-\beta \sum_{j=1}^{m} \frac{1}{j}\right) L(x), \quad x \in(0, \infty) .
$$


By setting $a=1$ in (3.7), one sees that for $x<0$, as $x \rightarrow-\infty$,

$$
F(x)=b(|x|) \frac{x^{m}}{m !}+\gamma L(|x|) \frac{x^{m}}{2 m !} \operatorname{sgn} x-\beta L(|x|) \frac{x^{m}}{m !} \sum_{j=1}^{m} \frac{1}{j}+o\left(|x|^{m} L(|x|)\right) .
$$

Since

$$
a^{-m} F(a x)-F(x)=\beta \log a \frac{x^{m}}{m !} L(x)+o\left(x^{m} L(x)\right), \quad x \rightarrow \infty,
$$

it is clear that for each $a>0$, we have

$$
b(a x)=b(x)+\beta \log a L(x)+o(L(x)), \quad x \rightarrow \infty .
$$

It is remarkable that, initially, no uniform condition on $a$ is assumed in (3.7). However, the proof of Theorem 3.2 forces this relation to hold uniformly for $a$ in compact subsets.

We are now ready to state the general structural theorem for negative integral degrees which now follows directly from Proposition 2.2, Theorem 3.1 and Theorem 3.2.

THEOREM 3.3. Let $f \in \mathcal{D}^{\prime}$ and $k$ be a positive integer. Then $f$ has the quasiasymptotic behavior in $\mathcal{D}^{\prime}$ at infinity,

$$
f(\lambda x)=\gamma \lambda^{-k} L(\lambda) \delta^{(k-1)}(x)+(-1)^{k-1}(k-1) ! \beta L(\lambda)(\lambda x)^{-k}+o\left(\lambda^{-k} L(\lambda)\right)
$$

if and only if there exist $m \in \mathbb{N}, m \geqslant k$, an associate asymptotically homogeneous function $b$ of degree 0 at infinity with respect to $L$ satisfying

$$
b(a x)=b(x)+\beta \log a L(x)+o(L(x)), \quad x \rightarrow \infty,
$$

for each $a>0$, and an $m$-primitive $F$ of $f$ which is continuous and satisfies

$$
\begin{aligned}
F(x)= & b(|x|) \frac{x^{m-k}}{(m-k) !}+\gamma L(|x|) \frac{x^{m-k}}{2(m-k) !} \operatorname{sgn} x \\
& -\beta L(|x|) \frac{x^{m-k}}{(m-k) !} \sum_{j=1}^{m-k} \frac{1}{j}+o\left(|x|^{m-k} L(|x|)\right)
\end{aligned}
$$

as $x \rightarrow \pm \infty$, in the ordinary sense. The last property is equivalent to

$$
\lim _{x \rightarrow \infty} \frac{(m-k) !\left(a^{k-m} F(a x)-(-1)^{m-k} F(-x)\right)}{x^{m-k} L(x)}=\gamma+\beta \log a,
$$

for each $a>0$.

It should be noticed that in (3.8) is not absolutely necessary to assume that the limit is of the form $\gamma+\beta \log a$. Indeed, we have the following corollary.

THEOREM 3.4. Let $f \in \mathcal{D}^{\prime}$. Then $f$ has quasiasymptotic at infinity of degree $-k, k \in\{1,2, \ldots\}$ if and only if there exists a continuous $m$-primitive $F$ of $f$, $m \geqslant k$, such that the following limit exists for each $a>0$,

$$
\lim _{x \rightarrow \infty} \frac{\left(a^{k-m} F(a x)-(-1)^{m-k} F(-x)\right)}{x^{m-k} L(x)}=I(a) .
$$


Proof. We show that $I(a)$ must be of the form $I(1)+\beta \log a$, for some constant $\beta$. We easily see that $I$ is measurable and satisfies $I(a b)=I(a)+I(b)-I(1)$, setting $h(x)=e^{I(x)-I(1)}$, one has that $h$ is positive, measurable and satisfies $h(a b)=$ $h(a) h(b)$, from where it follows $[\mathbf{1 1}]$ that $h(x)=x^{\beta}$, for some $\beta$, and so $I$ has the desired form.

We end this section with two remarks.

REMARK 3.1. Theorem 3.3 and Proposition 2.3 imply that if $f \in \mathcal{D}^{\prime}$ has quasiasymptotic of negative integral degree, then $f \in \mathcal{S}^{\prime}$. In fact, the structural theorem, Theorem 3.3, combined with Theorem 2.4 and [8, Theorem 3] imply that $f$ has the same quasiasymptotic in $\mathcal{S}^{\prime}$.

REMARK 3.2. The structural theorem for the quasiasymptotic of degree -1 implies at once the pointwise Fourier inversion formula for distributional point values given in $[\mathbf{1 2}, \mathbf{1 3}]$ and the formula for jump behavior of tempered distributions $[12, \mathbf{1 3}]$. For instance, in the case of $2 \pi$-periodic distributions, it states that $f(x)=$ $\sum_{-\infty}^{\infty} a_{n} e^{i n x}$ has the jump behavior at $x_{0}$

$$
\lim _{\varepsilon \rightarrow 0^{+}} f\left(x_{0}+\varepsilon x\right)=\gamma_{-} H(-x)+\gamma_{+} H(x) \text { in } \mathcal{D}^{\prime},
$$

if and only if there exists $m \in \mathbb{N}$ such that

$$
\lim _{x \rightarrow \infty} \sum_{-x \leqslant n<a x} a_{n} e^{i n x_{0}}=\frac{\gamma_{+}+\gamma_{-}}{2}+\frac{1}{2 \pi i}\left(\gamma_{+}-\gamma_{-}\right) \log a \quad(\mathrm{C}, m),
$$

for each $a>0$. Note that when $\gamma_{+}=\gamma_{-}$, we fall into the case of Estrada's characterization of Łojasiewicz point values of periodic distributions $[4,7]$.

\section{Other Results}

We end this article studying the following problem. Suppose that a distribution $f \in \mathcal{D}^{\prime}$ with support in $[0, \infty)$ has quasiasymptotic of degree $\alpha$ in the space $\mathcal{D}^{\prime}(0, \infty)$, that is, for each $\phi \in \mathcal{D}(0, \infty)$

$$
\lim _{\lambda \rightarrow \infty}\left\langle\frac{f(\lambda x)}{\lambda^{\alpha} L(\lambda)}, \phi(x)\right\rangle=\langle g(x), \phi(x)\rangle .
$$

What can we say about the quasiasymptotic properties of $f$ in $\mathcal{D}^{\prime}$ ?

We can apply the techniques of Sections 2 and 3 to give a complete answer to this question. The answer depends on $\alpha$.

Let us start with the case $\alpha>-1$. It is not difficult to show that $g$ must be of the form $C x_{+}^{\alpha} / \Gamma(\alpha+1)$, for some constant $C$. Next, Proposition 2.1 still holds replacing the space $\mathcal{D}^{\prime}$ by $\mathcal{D}^{\prime}(0, \infty)$ (actually this holds without the restriction $\alpha>-1)$. Hence, the same argument given in Theorem 2.3 applies here, but this time we only require the uniform convergence on $[1 / 2,2]$, and hence we can still conclude the existence of the integer such that (2.8) holds with the limit taken only as $x \rightarrow \infty$. Actually, because $\alpha>-1$, relation (2.8) holds for any $m$-primitive of 
$f$. Let $F$ be the $m$-primitive of $f$ supported on the interval $[0, \infty)$, then we have that

$$
F(x) \sim \frac{C x^{\alpha+m} L(x)}{\Gamma(\alpha+m+1)}, \quad x \rightarrow \infty,
$$

so we have that $F(\lambda x)=C L(\lambda)(\lambda x)_{+}^{\alpha+m} / \Gamma(\alpha+m+1)+o\left(\lambda^{\alpha+m} L(\lambda)\right)$ in the space $\mathcal{S}^{\prime}$, differentiating $m$-times, we obtain the following result.

THEOREM 4.1. Let $f \in \mathcal{D}^{\prime}$ be supported on $[0, \infty)$. If $f$ has quasiasymptotic behavior of degree $\alpha>-1$ in $\mathcal{D}^{\prime}(0, \infty)$, then it is a tempered distribution and has the same quasiasymptotic behavior in the space $\mathcal{S}^{\prime}$.

Suppose now that $\alpha<-1$ and $\alpha$ is not a negative integer. This case differs from the last case essentially in one point, we cannot conclude (2.8) for every $m$ primitive of $f$ but only for some of them. In any case, denoting again by $F$ the $m$-primitive (we keep $m>-\alpha-1$ ) of $f$ supported on $[0, \infty)$, we have that there exists a polynomial of degree at most $m-1$ such that

$$
F(x)-p(x) \sim \frac{C x^{\alpha+m} L(x)}{\Gamma(\alpha+m+1)}, \quad x \rightarrow \infty ;
$$

therefore,

$$
F(\lambda x)=\frac{C L(\lambda)(\lambda x)_{+}^{\alpha+m}}{\Gamma(\alpha+m+1)}+\sum_{j=0}^{m-1} a_{j}(\lambda x)_{+}^{j}+o\left(\lambda^{\alpha+m} L(\lambda)\right) \quad \text { as } \lambda \rightarrow \infty,
$$

in the space $\mathcal{S}^{\prime}$, for some constants $a_{0}, \ldots, a_{m-1}$. Thus, our arguments immediately imply the next theorem.

TheOREM 4.2. Let $f \in \mathcal{D}^{\prime}$ be supported on $[0, \infty)$. Suppose that

$$
f(\lambda x)=C L(\lambda) \frac{(\lambda x)_{+}^{\alpha}}{\Gamma(\alpha+1)}+o\left(\lambda^{\alpha} L(\lambda)\right) \quad \text { as } \lambda \rightarrow \infty \text { in } \mathcal{D}^{\prime}(0, \infty) .
$$

If $\alpha<-1$ and $\alpha$ is not a negative integer, then $f$ is a tempered distribution. Moreover, there exist constants $a_{0}, a_{1}, \ldots, a_{n}(n<-\alpha-1)$ such that

$$
f(\lambda x)=C L(\lambda) \frac{(\lambda x)_{+}^{\alpha}}{\Gamma(\alpha+1)}+\sum_{j=0}^{n} a_{j} \frac{\delta^{(j)}(x)}{\lambda^{j+1}}+o\left(\lambda^{\alpha} L(\lambda)\right) \quad \text { as } \lambda \rightarrow \infty \text { in } \mathcal{S}^{\prime} .
$$

When $\alpha=-k, k$ being a positive integer, the distribution $g$ in (4.1) must have the form $C x^{-k} \in \mathcal{D}^{\prime}(0, \infty)$, for some constant $C$; these distributions are homogeneous as elements of $\mathcal{D}^{\prime}(0, \infty)$, but they do not have homogeneous extensions to $\mathcal{D}$. The behavior of $f(\lambda x)$ as $\lambda \rightarrow \infty$ in $\mathcal{S}^{\prime}$ is described in the next theorem.

TheOREM 4.3. Let $f \in \mathcal{D}^{\prime}$ be supported on $[0, \infty)$. Suppose that

$$
f(\lambda x)=C L(\lambda) \frac{H(x)}{(\lambda x)^{k}}+o\left(\frac{L(\lambda)}{\lambda^{k}}\right) \quad \text { as } \lambda \rightarrow \infty \text { in } \mathcal{D}^{\prime}(0, \infty),
$$


where $k$ is a positive integer. Then $f$ is a tempered distribution and there exist an associate asymptotically homogeneous function b satisfying

$$
b(a x)=b(x)+\frac{(-1)^{k-1}}{(k-1) !} C L(x) \log a+o(L(x)), \quad x \rightarrow \infty,
$$

for each $a>0$, and constants $a_{0}, a_{1}, \ldots, a_{k-1}$ such that

$$
f(\lambda x)=C \frac{L(\lambda)}{\lambda^{k}} \operatorname{Pf}\left(\frac{H(x)}{x^{k}}\right)+\frac{b(\lambda)}{\lambda^{k}} \delta^{(k-1)}(x)+\sum_{j=0}^{k-1} a_{j} \frac{\delta^{(j)}(x)}{\lambda^{j+1}}+o\left(\frac{L(\lambda)}{\lambda^{k}}\right)
$$

as $\lambda \rightarrow \infty$ in $\mathcal{S}^{\prime}$.

Proof. For each $n \in \mathbb{N}$, let $F_{n}$ denote the $n$-primitive of $f$ with support in $[0, \infty)$. Set $C_{1}=(-1)^{k-1} C /(k-1)$ !. Adapting the arguments of Section 3 and reasoning as in the previous two cases, we obtain the existence of a positive integer $m>k$ such that $F_{m}$ is continuous and

$$
F_{m}(x)=b_{1}(x) \frac{x^{m-k}}{(m-k) !}-C_{1} L(x) \frac{x^{m-k}}{(m-k) !} \sum_{j=1}^{m-k} \frac{1}{j}+p_{m-1}(x)+o\left(x^{m-k} L(x)\right),
$$

$x \rightarrow \infty$, where $b_{1}$ is a locally integrable associate asymptotically homogeneous function satisfying (4.2) and $p_{m-1}$ is a polynomial of degree at most $m-1$. Throwing away the irrelevant terms of the polynomial $p_{m-1}$ and using Theorem 2.4 , we obtain the following asymptotic expansion as $\lambda \rightarrow \infty$ in the space $\mathcal{S}^{\prime}$,

$$
\begin{aligned}
F_{m}(\lambda x)= & b_{1}(\lambda) \frac{(\lambda x)_{+}^{m-k}}{(m-k) !}+C_{1} \lambda^{m-k} L(\lambda) l_{m-k}(x) H(x) \\
& +\sum_{j=0}^{k-1} a_{j} \frac{(\lambda x)_{+}^{m-j-1}}{(m-j-1) !}+o\left(\lambda^{m-k} L(\lambda)\right) .
\end{aligned}
$$

Differentiating $(m-k)$-times this expansion, we have that

$$
F_{k}(\lambda x)=b_{1}(\lambda) H(x)+C_{1} L(\lambda) H(x) \log x+\sum_{j=0}^{k-1} a_{j} \frac{(\lambda x)_{+}^{k-j-1}}{(k-j-1) !}+o(L(\lambda)) .
$$

The formulas [5, p. 68],

$$
\begin{aligned}
\frac{d}{d x}(H(x) \log x) & =\operatorname{Pf}\left(\frac{H(x)}{x}\right) \\
\frac{d}{d x}\left(\operatorname{Pf}\left(\frac{H(x)}{x^{n}}\right)\right) & =-n \operatorname{Pf}\left(\frac{H(x)}{x^{n+1}}\right)+\frac{(-1)^{n} \delta^{(n)}(x)}{n !}
\end{aligned}
$$

imply that

$$
\frac{d^{k-1}}{d x^{k-1}}\left(\operatorname{Pf}\left(\frac{H(x)}{x}\right)\right)=(-1)^{k-1}(k-1) ! \operatorname{Pf}\left(\frac{H(x)}{x^{k}}\right)-\delta^{(k-1)}(x) \sum_{j=1}^{k-1} \frac{1}{j} .
$$


Hence, differentiating (4.4) $k$-times, one has (4.3) with

$$
b(x)=b_{1}(x)+\frac{(-1)^{k} C}{(k-1) !}\left(\sum_{j=1}^{k-1} \frac{1}{j}\right) L(x) .
$$

\section{References}

[1] J. M. Ash, P. Erdős, L. A. Rubel, Very slowly varying functions, Aequationes Math. 10 (1974), 1-9.

[2] R. Bojanić, J. Karamata, On a class of functions of regular asymptotic behavior, Math. Res. Centre, U.S. Army, Madison, Wis., Tech. Summary Rep. No. 436 (1963).

[3] Yu. N. Drozhzhinov, B.I. Zav'yalov, Asymptotically homogeneous generalized functions in a spherical representation and some applications, Dokl. Akad. Nauk 405 (2005), 18-21 (in Russian).

[4] R. Estrada, Characterization of the Fourier series of distributions having a value at a point, Proc. Amer. Math. Soc. 124 (1996), 1205-1212.

[5] R. Estrada, R. P. Kanwal, A distributional approach to Asymptotics: Theory and Applications, Second Edition, Birkhäuser, Boston, 2002.

[6] H. Korevaar, T. van Aardenne-Ehrenfest, N. G. de Bruijn, A note on slowly oscillating functions, Nieuw. Arch. Wisk. 23 (1949), 77-88.

[7] S. Łojasiewicz, Sur la valuer et la limite d'une distribution en un point, Studia Math. 16 (1957), 1-36.

[8] S. Pilipović, Some properties of the quasiasymptotic of Schwartz distributions. I. Quasiasymptotic at $\pm \infty$, Publ. Inst. Math. (Beograd) $\mathbf{5 7}$ (1988), 125-130.

[9] S. Pilipović, On the quasiasymptotic of Schwartz distributions, Math. Nachr. 137 (1988), $19-25$.

[10] L. Schwartz, Théorie des Distributions, Hermann, Paris, 1966.

[11] E. Seneta, Regularly Varying Functions, Springer-Verlag, Berlin, 1976.

[12] J. Vindas, R. Estrada, Distributionally regulated functions, Studia Math. 181 (2007), 211236 .

[13] J. Vindas, R. Estrada, Distributional Point Values and Convergence of Fourier Series and Integrals, J. Fourier. Anal. Appl. 13 (2007), 551-576.

[14] J. Vindas, R. Estrada, On the Order of Summability of the Fourier Inversion Formula, preprint.

[15] J. Vindas, S. Pilipović, Structural Theorems for Quasiasymptotics of Distributions at the Origin, Math. Nachr., in press.

[16] V.S. Vladimirov, Yu. N. Drozhzhinov, B. I. Zav'yalov, Tauberian theorems for generalized functions in a scale of regularly varying functions and functionals, dedicated to Jovan Karamata, Publ. Inst. Math. (Beograd) 71 (2002), 123-132 (in Russian).

[17] V.S. Vladimirov, Yu. N. Drozzinov, B. I. Zavialov, Tauberian Theorems for Generalized Functions, Kluwer, Maine, 1988.

[18] B. I. Zavialov, Automodel asymptotics of electromagnetic form factors and the behavior of their Fourier transforms, Teoret. Mat. Fiz. 17 (1973), 178-188 (in Russian).

Department of Mathematics

Louisiana State University

Baton Rouge, LA 70803

U.S.A.

jvindas@math.1su.edu 